\title{
Fundamental Study for Optical Transillumination Imaging of Arteriovenous Fistula
}

\author{
Hideaki KamiYama, ${ }^{*}$ Masataka Kitama, ${ }^{* *}$ \# Hisae O. Shimizu, ${ }^{* * *}$ Masaji Yamashita, ${ }^{* * *}$ Yohichiro KoJIma, ${ }^{\dagger}$ \\ Koichi SHIMIZU ${ }^{\dagger \dagger}$
}

\begin{abstract}
An arteriovenous (AV) fistula is commonly constructed in patients who need dialysis treatment. Daily monitoring of the AV fistula is crucially important to maintain its correct function. We developed a new technique using a safe and simple instrument for noninvasive monitoring of the AV fistula. Using this technique, we can observe and analyze the blood vessel condition using transillumination imaging with near-infrared (NIR) light. For an AV fistula in the forearm, we illuminate NIR light from both sides of the arm. Then the transillumination image is captured using a camera placed above the forearm. An imaging system was developed to validate the proposed technique. It was tested with model phantoms that simulate the blood vessels of the human adult forearm. Severe blurring of the image caused by strong scattering in the interstitial tissue can be eliminated by deconvolution with a point spread function of the blur. This process clarified the blood vessel image and facilitated measurement of its inner diameter. After confirming the accuracy of the system, we attempted measurement of localized stenosis. Results show that we can detect stenotic portions as small as $2 \mathrm{~mm}$ in diameter and $2 \mathrm{~mm}$ in length. The detectability of non-coaxial stenosis was also confirmed. These results verified the feasibility and applicability of the proposed technique for non-invasive daily monitoring of AV fistulae.
\end{abstract}

Keywords: AV fistula, NIR light, noninvasive, PSF deconvolution, transillumination imaging.

Adv Biomed Eng. 10: pp. 1-10, 2021.

\section{Introduction}

Patients with end-stage renal failure need to undergo blood purification by dialysis treatment. In recent years, the number of chronic dialysis patients has been increas-

Received on June 12, 2020; revised on October 29, 2020; accepted on November 17, 2020

* Graduate School of Engineering, Division of Engineering, Hokkaido University of Science, Sapporo, Japan.

*** Department of Radiological Technology, Faculty of Health Sciences, Hokkaido University of Science, Sapporo, Japan.

**** Department of Clinical Engineering, Faculty of Health Sciences, Hokkaido University of Science, Sapporo, Japan.

${ }^{\dagger}$ Department of Electrical and Electronic Engineering, Faculty of Engineering, Hokkaido University of Science, Sapporo, Japan.

${ }^{\dagger \dagger}$ Graduate School of Information, Production and Systems, Waseda University, Kitakyushu, Japan.

\# 7-Jo 15-4-1 Maeda, Teine, Sapporo, Hokkaido 006-8585, Japan. E-mail: kitama@hus.ac.jp

\section{(c) BY} Copyright: (92021 The Author(s). This is an open access article distributed under the terms of the Creative Commons BY 4.0 International (Attribution) License (https:// creativecommons.org/licenses/by/4.0/legalcode), which permits the unrestricted distribution, reproduction and use of the article provided the original source and authors are credited. ing because of the rapid diffusion of medical technology throughout economically developing countries. These patients must undergo treatment a few times a week for about 4 hours each. Moreover, they need vascular access with sufficient blood flow to maintain extracorporeal circulation at a high flow rate. Easy needling is also necessary in practice. To meet these requirements, an arteriovenous (AV) fistula is usually constructed in the patient's forearm. After the artery and the vein are anastomosed subcutaneously, a needle is inserted into the vein $[1,2]$. However, in the AV fistula, unfavorable stress is exerted on the venous wall because of the non-physiological blood flow. Moreover, the repeated needle puncture necessary for every dialysis imparts external stress on the blood vessel. Consequently, the vein wall thickens, leading to stenosis [3]. Therefore, detecting changes in inner diameter of the vein in the AV fistula is important.

Currently, visual inspection, auscultation, and palpation are commonly practiced, but they can yield only secondary information. Moreover, they are no more than subjective assessments. X-ray angiography can provide a quantitative inner diameter, but it is unsuitable for daily management because of radiation issues and equipment size. Ultrasound imaging is another alternative. In addition to the image, it can provide blood flow by Doppler 
measurements. Nevertheless, some practical difficulties persist such as noisy images, poor spatial resolution, and dependence on the examiner's skill. Due to the recent progress in homecare medicine, dialysis at home is increasing steadily. Given this trend, traditional inspection methods that rely on the experience and skills of medical staff are no longer appropriate for home dialysis. Strong demand exists today for the implementation of non-invasive and quantitative management of AV fistulae.

The use of light is one candidate for non-invasive measurement of animal bodies. Extensive studies of such methods have been conducted [4]. We propose the application of near-infrared (NIR) transillumination imaging for the management of AV fistulae. Using this technique, we visualize the subcutaneous AV fistula and measure the blood vessel inner diameter. To realize early detection of stenosis, a test system was constructed to monitor the AV fistula conditions. Using this system, we can achieve quantitative blood vessel measurements without using X-rays. Using the safe and simple instrumentation, daily continuous monitoring of the fistula condition becomes possible.

\section{Imaging and Measuring Methods}

\subsection{Optical transillumination imaging}

Although optical imaging is useful for noninvasive measurement of animal bodies, visible light is strongly attenuated when propagated through the animal body. However, NIR light of 700-1200 nm wavelength (known as the biological window) has low attenuation through animal tissues. Therefore, a transillumination image is obtainable using NIR light. Because the major light absorber in human body tissue is hemoglobin in the blood, changes in blood vessel diameter are detectable in the transillumination image. Oxyhemoglobin $(\mathrm{HbO})$ and deoxyhemoglobin $(\mathrm{Hb})$ show different absorption spectra in the NIR wavelength. This difference is useful to measure the oxygen saturation of the blood. However, this dependence of light absorption on oxygen saturation is unfavorable for quantitative analyses of blood vessel diameter from the transillumination image. Therefore, we chose $810 \mathrm{~nm}$ as the wavelength, close to the isosbestic point at which the absorbance of $\mathrm{HbO}$ and $\mathrm{Hb}$ are equal. Using NIR light of this wavelength, one can visualize the blood vessel in the AV fistula and measure the blood vessel diameter irrespective of changes in blood oxygenation.

\subsection{Blood vessel imaging with scattered light}

Various reports have described blood vessel transillumination imaging $[5,6]$. They can be classified into two types: reflection and transmission types. A light source is placed on the same side of the imaging object, and an

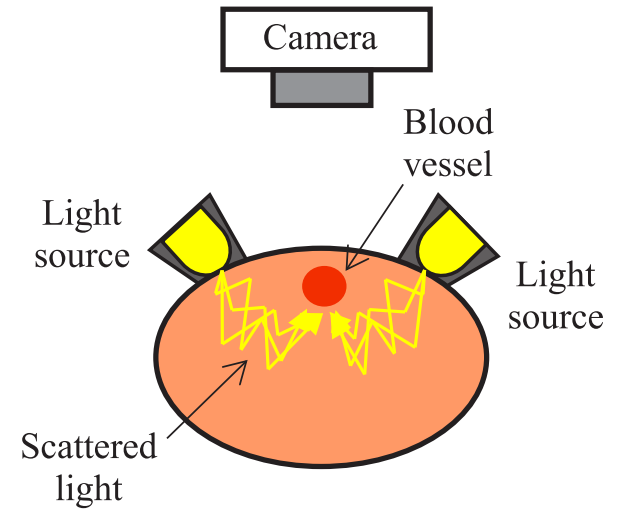

Fig. 1 Principle of the proposed technique.

imaging device on the opposite side. For example, the reflection type is used to visualize the surface vein network $[7,8]$, and the transmission type is used for finger vein authentication $[9,10]$. The salient advantage of the reflection type is its applicability to thick body parts through which the light cannot pass. However, it is unsuitable for accurate subsurface measurement. For accurate transillumination measurements, homogeneous illumination and distortionless image projection are required. In reflection type imaging of a human body, these requirements are often difficult to satisfy because of the direct illumination and the strong surface scattering at the irregularly shaped body surface. By contrast, for the transmission type, illumination of the target blood vessel from the backside is almost homogenized by strong scattering in the backing tissue. Surface scattering also poses little difficulty. However, a severe shortcoming of the transmission type is light attenuation through the body tissue. The applicability is limited to the thin parts of a human body. In adults, it is applicable to a finger, limited areas of a hand, or a foot.

The AV fistula is commonly constructed subcutaneously in the forearm at a depth of approximately $1.0 \mathrm{~mm}$. Sufficient light intensity is difficult to obtain through the thickness of the arm [11-16]. We used a scattering illumination technique to alleviate this difficulty: it illuminates both sides of the forearm. Figure 1 presents the principle of this technique. The subcutaneous blood vessels are illuminated by light that is scattered by body tissues. It works as back-illumination from a backing light diffuser for subcutaneous blood vessels. By virtue of this side-illumination, we can ensure sufficient light intensity for transillumination imaging, even in an adult arm.

\section{Imaging and Measurement System}

\subsection{Measurement system using scattered light}

An experimental system was developed to validate of the proposed technique. Figure 2(a) depicts an outline of 


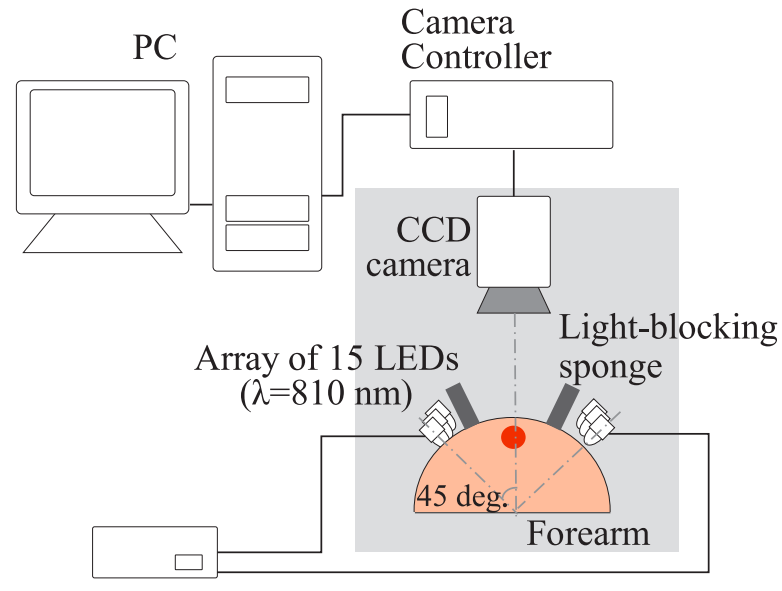

LED Power supply

(a) Outline of developed system

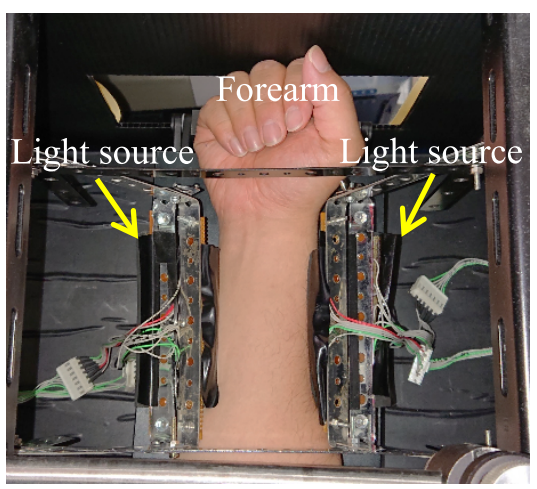

(b) Appearance of applicator part

Fig. 2 Transillumination imaging system to visualize blood vessels of the human forearm.

the developed system.

Two linear arrays of LEDs were placed at either side of the forearm. Each array consisted of 15 LEDs $(1.4 \times$ $15 \mathrm{~mW}$ optical power, L810-03AU; Ushio Opto Semiconductors, Inc.). The optical axes of LEDs were oriented towards the center of the hemispherical cross section of the forearm phantom. The angle of the LEDs axis was 45 degrees from the optical axis of the camera. Because the output of the diffuse light from the phantom is not very sensitive to this angle, 45 degrees was chosen to ensure practical convenience of the measurement system.

A CCD camera (C4742-95; Hamamatsu Photonics K.K.) was placed above the forearm to receive the transmitted light from the forearm. The LEDs were covered with a black light-blocking sponge to prevent light propagation along the body surface and to prevent direct light leakage toward the camera. This structure obviates the need to press the light source firmly against the body surface. We can record a transillumination image with light contact of the sponge to the body surface. This ease of recording is an important benefit of noninvasive monitor-

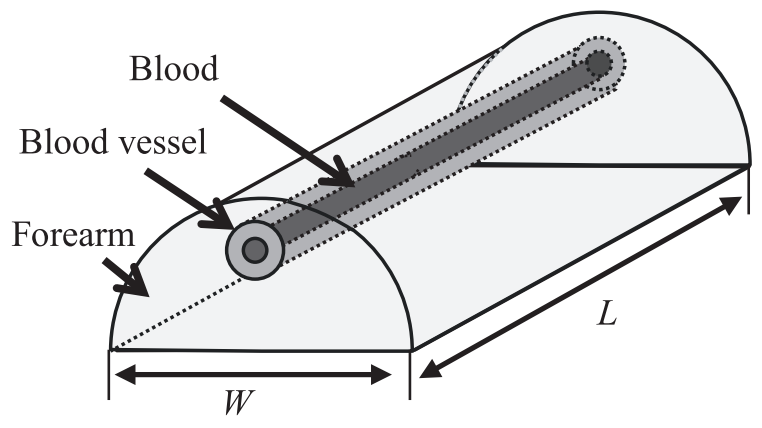

(a) Structure of model phantom

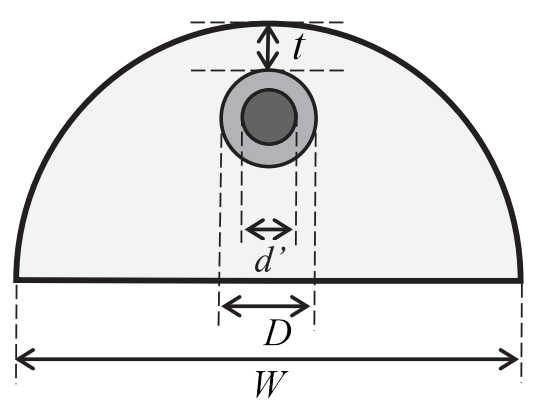

(b) Cross-sectional view of model phantom

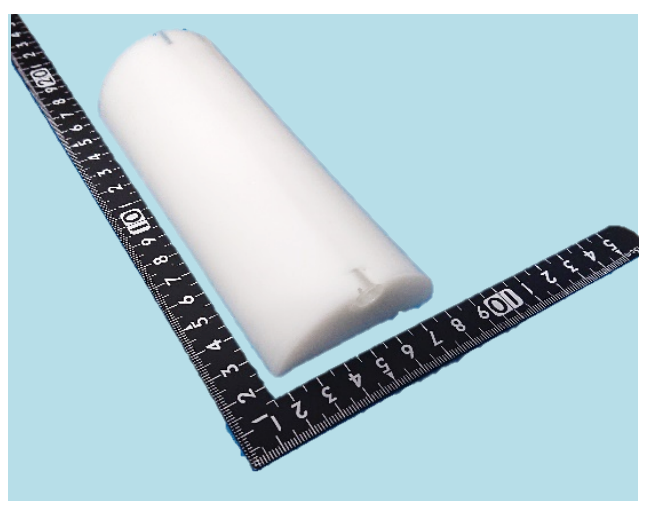

(c) Appearance of phantom

Fig. 3 Structure and appearance of model phantom to simulate blood vessel in human forearm: $W=65.0 \mathrm{~mm}$, $L=150.0 \mathrm{~mm}, D=7.0 \mathrm{~mm}, d^{\prime}=5.0 \mathrm{~mm}, t=1.0 \mathrm{~mm}$.

ing of the AV fistula. Figure 2(b) portrays the appearance of the applicator part of the developed system.

\subsection{Model phantom of a human forearm}

To examine the blood vessel measurement accuracy, a model phantom with a known structure was used. Figure 3 portrays the model phantom structure used to simulate the major vein in a human adult forearm. The phantom is a solid half-cylinder. At both ends of the cylinder are dents formed during the manufacturing process. Because the measurement area is limited to the central $10.0 \mathrm{~mm}$ square for a length of $150 \mathrm{~mm}$ along the cylindrical surface, these dents exert no effect. 
The absorption coefficient $\left(\mu_{\mathrm{a}}\right)$ and the reduced scattering coefficient $\left(\mu_{\mathrm{s}}^{\prime}\right)$ for the blood, the blood vessel wall and the surrounding tissue models were, respectively, $\left(\mu_{\mathrm{a}}=0.45 \mathrm{~mm}^{-1}, \mu_{\mathrm{s}}{ }^{\prime}=0.39 \mathrm{~mm}^{-1}\right),\left(\mu_{\mathrm{a}}=0.15 \mathrm{~mm}^{-1}\right.$, $\left.\mu_{\mathrm{s}}^{\prime}=1.50 \mathrm{~mm}^{-1}\right)$, and $\left(\mu_{\mathrm{a}}=0.002 \mathrm{~mm}^{-1}, \mu_{\mathrm{s}}^{\prime}=\right.$ $1.20 \mathrm{~mm}^{-1}$ ) [12-14]. The blood vessel outer and inner diameters were $7.0 \mathrm{~mm}$ and $5.0 \mathrm{~mm}$, respectively. The blood vessel model depth was $t=1.0 \mathrm{~mm}$, which is the usual depth of the AV fistula in patients [1]. The base material was epoxy resin (Z-1; Nissin Resin Co., Ltd.). Black ink (INK-350-B; Pilot Co.) and titanium oxide (53140-1250; Junsei Chemical Co., Ltd.) were mixed to control absorption and scattering, respectively.

\section{IMAGE PROCESSING FOR WALL DE- TECTION}

\subsection{Edge enhancement by unsharp masking}

Using the proposed technique, we evaluated the blood vessel inner diameter in the transillumination image of the forearm. The blood vessel image was severely blurred by strong light scattering at the interstitial tissues between the blood vessel and the skin surface. First, we applied a common image processing filter for edge enhancement, or an unsharp mask. By this technique, an original transillumination image $(1024 \times 1024$ pixels $)$ is blurred intentionally by convolution of a $1024 \times 1024$ pixel square matrix. The original image is divided by the blurred image, yielding a sharper image. Figure 4 presents an example of this filter technique. As expected, the image blur was suppressed effectively. Nevertheless, the edge of the vessel wall was insufficiently clarified for accurate edge detection.

\subsection{Scattering suppression by PSF deconvolution}

The image blur is regarded as convolution of a clear image with the point spread function (PSF), which characterizes the blur for a point image. The PSF for a turbid medium such as animal tissue was derived theoretically, and its validity was confirmed through experimentation [17]. Therefore, we can deblur the transillumination image by deconvolution using this PSF. The PSF is shown below.

$$
\begin{aligned}
\operatorname{PSF}(\rho, d)= & C\left\{\left(\mu_{s}^{\prime}+\mu_{a}\right)+\left(\kappa_{d}+\frac{1}{\sqrt{\rho^{2}+d^{2}}}\right)\right. \\
& \left.\times \frac{d}{\sqrt{\rho^{2}+d^{2}}}\right\} \frac{\exp \left(-\kappa_{d} \sqrt{\rho^{2}+d^{2}}\right)}{\sqrt{\rho^{2}+d^{2}}}
\end{aligned}
$$

In that equation, $k_{d}{ }^{2}=3 \mu_{\mathrm{a}}\left(\mu_{\mathrm{s}}{ }^{\prime}+\mu_{\mathrm{a}}\right)$, and $\rho, d$ and $C$ denote the horizontal distance from an absorbing object, the vertical depth of the absorber and a constant, respectively. In the application described in the following sections, the typical values are $\rho=-10$ to $10 \mathrm{~mm}, d=$ $4.5 \mathrm{~mm}, \mu_{\mathrm{a}}=0.002 \mathrm{~mm}^{-1}$, and $\mu_{\mathrm{s}}^{\prime}=1.20 \mathrm{~mm}^{-1}$.
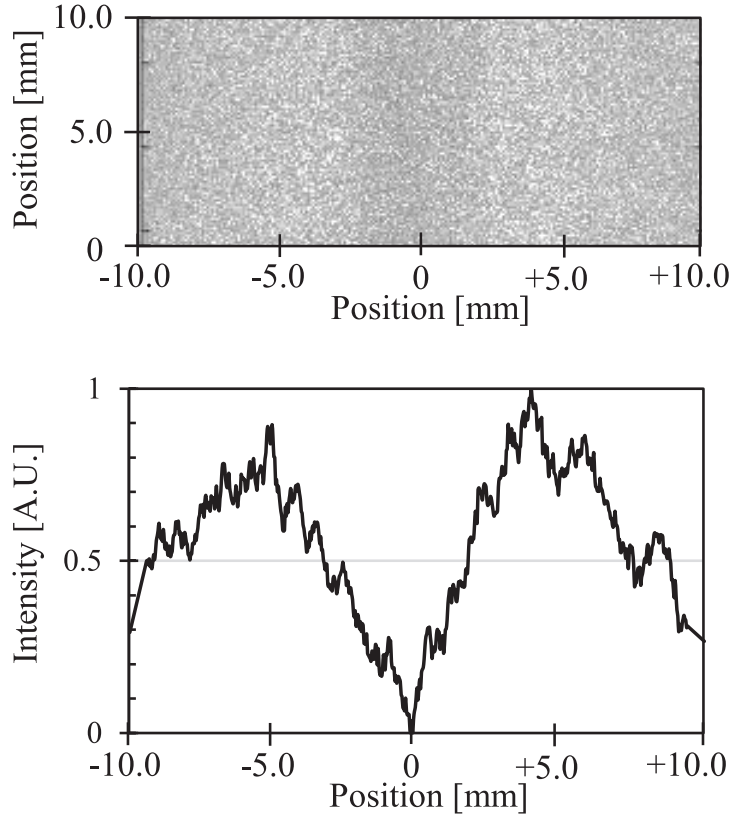

(a) Filtered image and intensity profile along central horizontal line
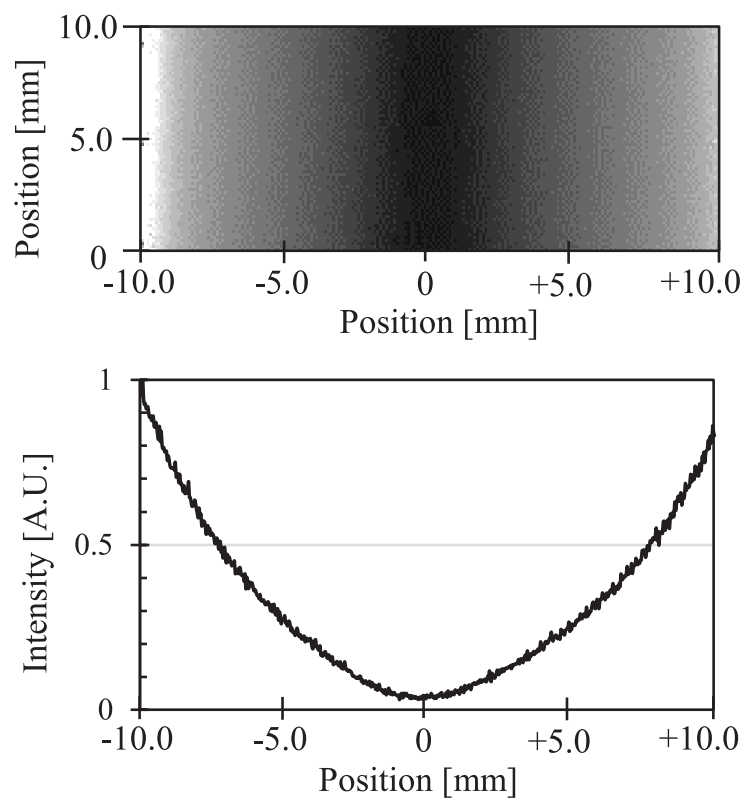

(b) Original image and intensity profile along central horizontal line

Fig. 4 Deblurring by conventional image processing.

This PSF is derived for the image of a point light source in a turbid medium, but the blood vessel image is observed as a shadow of the light absorbing material, or the blood. If one regards the shadow image as a collection of light absorbing points, then the same equation is useful for deblurring [12]. The PSF is derived for the flat surface of a turbid medium. Because the PSF is applied to the image around a blood vessel typically in a $10 \times$ $10 \mathrm{~mm}$ square on the surface of a cylinder $65 \mathrm{~mm}$ in di- 
ameter, the surface can be approximated as a flat surface. During deconvolution of a $1024 \times 1024$ pixel image with the PSF of the same size, the image is padded with zero data for 1023 pixels vertically and horizontally.

Figure 5 presents an example of the deblurring effect. Severe blurring in Fig. 5(a) is suppressed effectively. The boundary between the blood and the vessel wall is clarified. From this deblurred image, the inner diameter of the blood vessel can be evaluated. We used this PSF deconvolution technique in this study to analyze the transillumination image of the blood vessel in an AV fis-
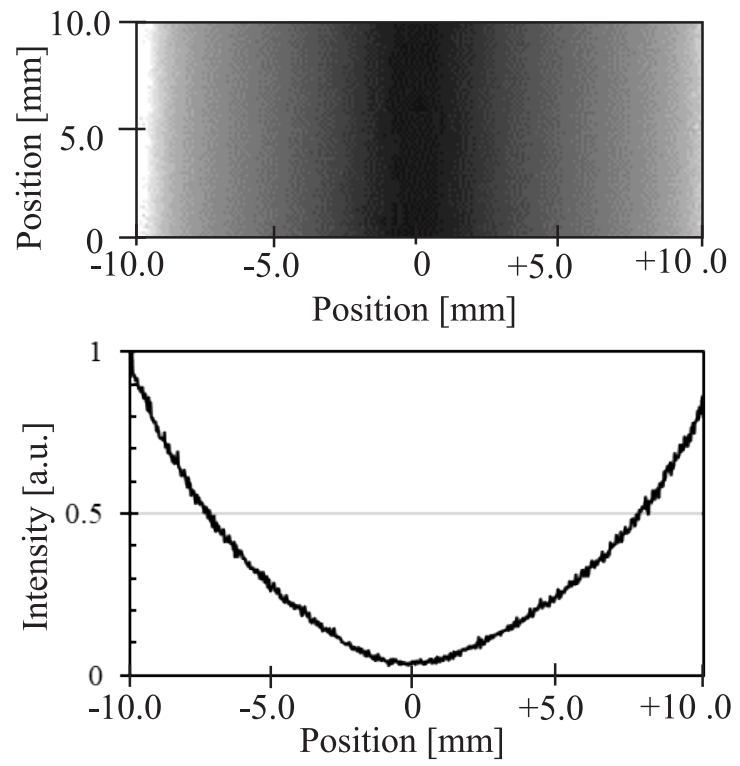

(a) Original image and intensity profile along central horizontal line
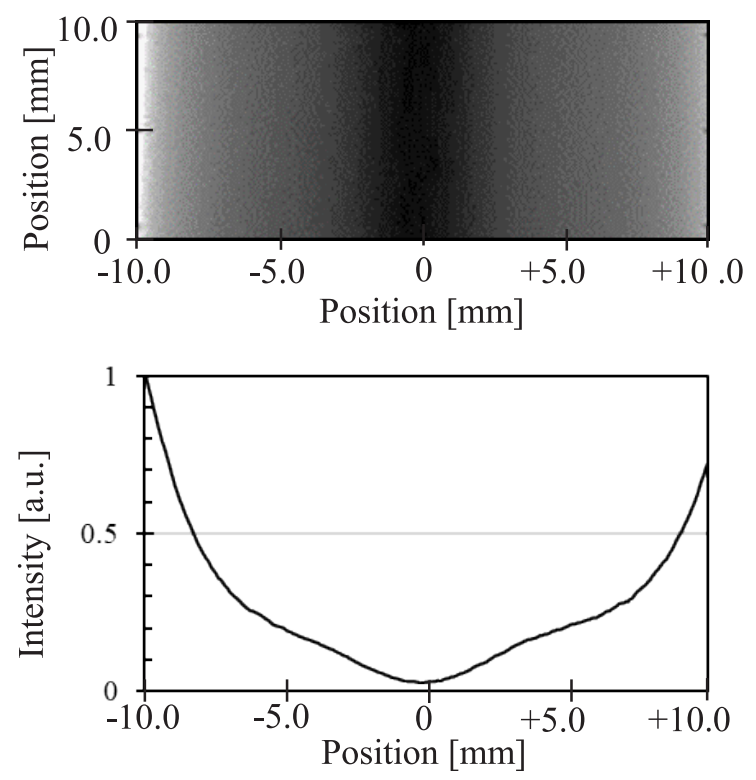

(b) Deconvolved image with PSF and intensity profile along central horizontal line

Fig. 5 Deblurring by PSF derived for suppression of scattering effect. tula.

\subsection{Wall detection by differentiation}

An appropriate deblurring technique can support clearer transillumination imaging. However, identification of the boundary between the blood and the vessel wall is still subjective. The method should be objective and automated, particularly for home dialysis. We propose a technique to analyze the blood vessel image intensity profile. Figure 6 presents the principle of inner wall detection. For demonstration with less noise, the scattering coefficient of the phantom was reduced to $1 / 10$ of the common value. After deblurring of the transillumination image, the intensity profile is analyzed across the blood vessel. As depicted in Fig. 6(a), discontinuities in the profile are apparent at the tissue-vessel wall and the vessel wallblood interfaces. The profile gradient is calculated by spatial differentiation of the intensity profile. Figure 6(b) shows the distribution of the absolute value of the differentiation. With this edge enhancement, the blood boundary can be detected and the blood vessel inner diameter can be measured objectively. For this study, the horizontal intensity profiles across the blood vessel image were sampled at five vertical positions with 5-pixel interval and were averaged to suppress image noise effects.

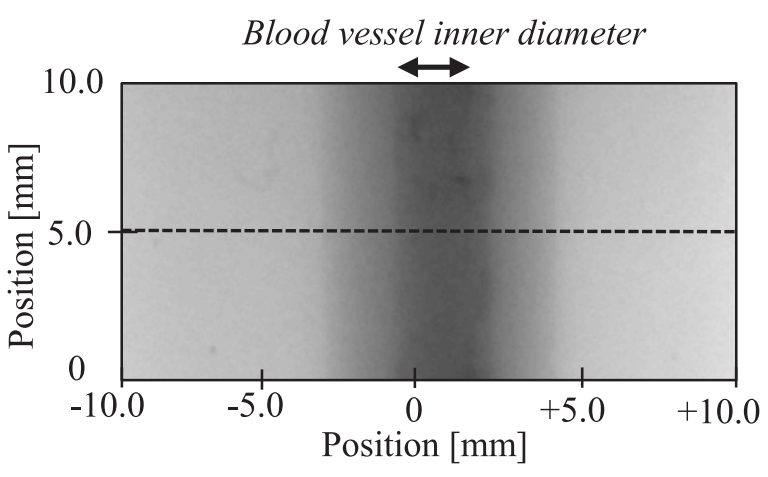

(a) Deconvoluted image

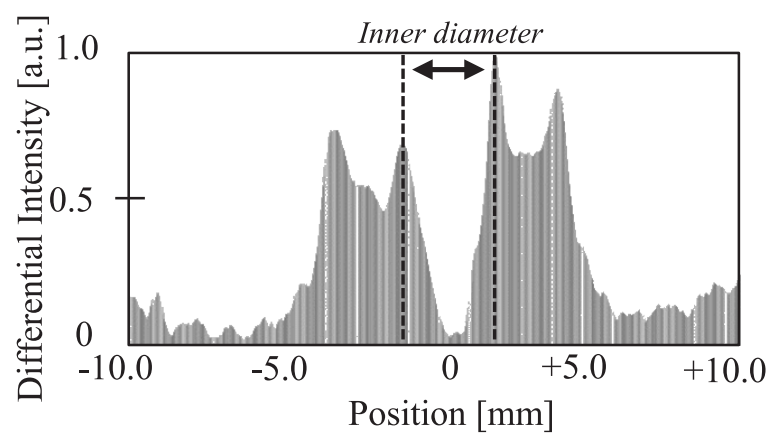

(b) Differentiated profile

Fig. 6 Principle of vessel wall detection by intensity profile differentiation: outer diameter $D=7.0 \mathrm{~mm}$, inner diameter $d^{\prime}=3.0 \mathrm{~mm}$. 


\section{EXPERIMENTAL TESTS OF PROPOSED TECHNIQUE}

\subsection{Detection of diameter change}

The feasibility of the proposed technique was tested through experimentation. First, the capability of the technique to detect blood vessel diameter changes was examined. We prepared model phantoms as depicted in Fig. 3, modeling blood vessels of three different inner diameters. The vessel outer diameter was kept constant at $7 \mathrm{~mm}$ in all three models. Figure 7 presents the measurement results. From the original image, we detected the existence of the blood vessel, but not the vessel wall or the vessel-blood interface, even after differentiation. By contrast, the interface was clarified by PSF deconvolution, and the inner diameter was estimated to within $30 \%$ error.

\subsection{Detection of localized change}

One important objective of this study is detection of stenosis. A stenosis often occurs only at a portion of the blood vessel. When the length of stenosis along the blood vessel is short, its detection becomes difficult. To assess the effectiveness of the proposed technique to detect such a stenosis, we produced a model phantom to simulate the different stages of stenosis in the AV fistula. Figure 8 portrays the phantom structure. The 5-mm inner diameter blood vessel model was narrowed partially. The length of the narrow portion was $2.0-4.0 \mathrm{~mm}$; the diameter was varied from 3.0 to $1.5 \mathrm{~mm}$ to simulate the progression of stenosis.

Figure 9 portrays results of transillumination imaging of the stenotic model phantom. White dots show the

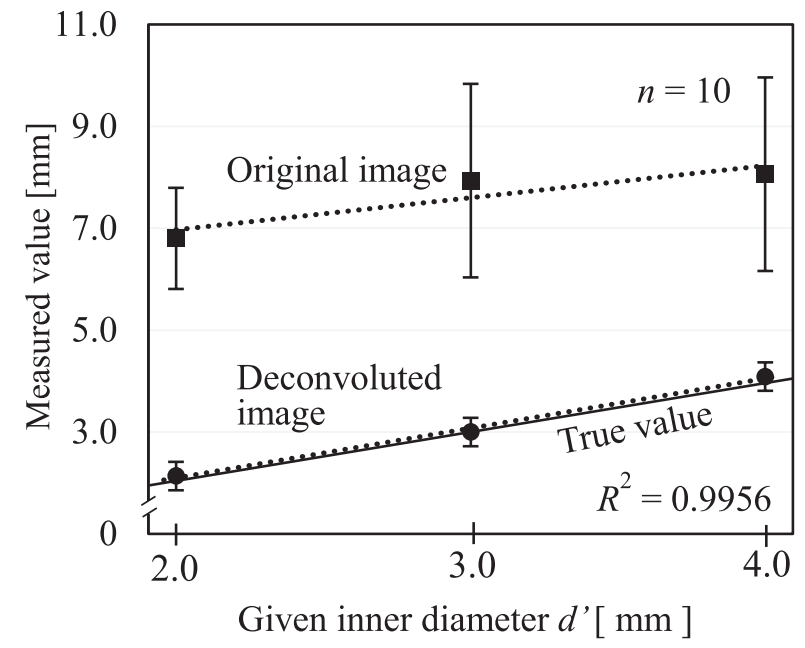

Fig. 7 Inner diameter of blood vessel model in original and deblurred images: Error bars show $m \pm \sigma$, where $m$ and $\sigma$ denote the mean and standard deviation of repeated measurements.

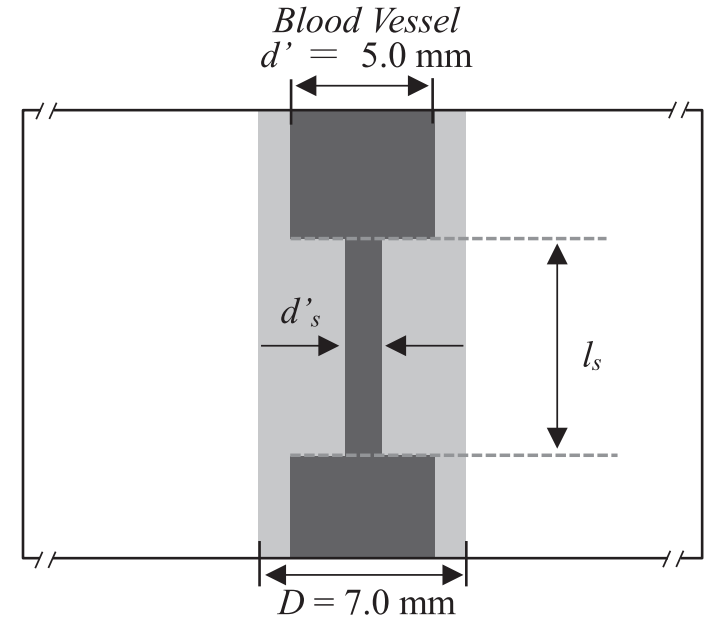

Fig. 8 Cross section of phantom structure to simulate coaxial localized stenosis: $d_{s}$, inner diameter of stenotic portion; $l_{s}$, longitudinal length of stenotic portion; intact blood vessel inner diameter $d^{\prime}=5.0 \mathrm{~mm}$; blood vessel outer diameter $D=7.0 \mathrm{~mm}$; blood vessel depth $t=1.0 \mathrm{~mm}$.

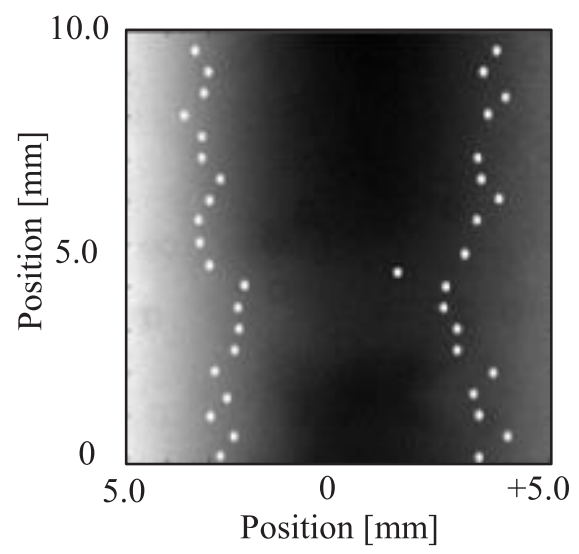

(a) Original image and detected boundary

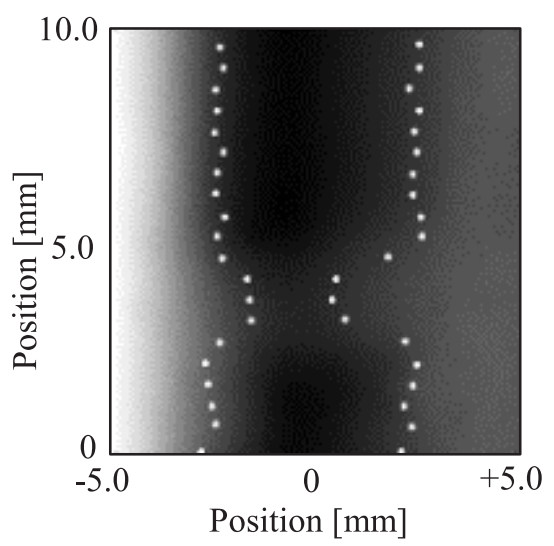

(b) Deblurred image and detected boundary

Fig. 9 Detection of stenosis in transillumination imaging: White dots show the boundary detected in horizontal intensity profile: outer diameter $D=7.0 \mathrm{~mm}$; inner diameter $d^{\prime}=5.0 \mathrm{~mm}$; inner diameter of stenotic part $d_{s}{ }_{s}=2.0 \mathrm{~mm}$; length of stenotic part $l_{s}=2.0 \mathrm{~mm}$. 
positions identified as the vessel wall-blood boundary. With appropriate deblurring, the stenotic part was detected well. Using these images, we evaluated the degree of the stenosis. Figure 10 presents results of inner diameter measurements in the stenosis model. Decrease in inner diameter of as short as $2.0 \mathrm{~mm}$ was detectable within the stenotic portion.

The measured diameter was overestimated compared to the true value. The estimation error for the typical diameter (more than $2.0 \mathrm{~mm}$ ) was less than $35 \%$. Narrower inner diameter and shorter stenotic length exacerbated this overestimation. This fact can be explained by the difference in the degree of scattering blur. The albedos of the blood and the vessel wall are 0.46 and 0.91 . The latter is a highly scattering-dominant material. Therefore, when the ratio of the vessel wall in the image ROI becomes large, the scattering blur becomes large. In the case of narrower inner diameter and shorter stenotic length, this ratio becomes larger. The scattering blur becomes more severe. This result may cause overestimation of the blood vessel diameter.

This result suggests that we can detect the stenotic change of the inner diameter if the stenosis is more than $2.0 \mathrm{~mm}$. It also suggests that there is no change in accuracy in the estimation of inner diameter when the stenosis length exceeds $3.0 \mathrm{~mm}$. With more effective scattering suppression, further improvement of accuracy is expected.

According to the guideline, the inner diameter of the vein is expected to be more than $2 \mathrm{~mm}$ when constructing the AV fistula [18]. Because the diameter becomes larger due to vascular dilation during dialysis, a measurement as small as $2 \mathrm{~mm}$ diameter is still useful for stenosis detection. In clinical practice, observation of a stenosis around $3 \mathrm{~mm}$ diameter is useful for the decision of surgical intervention [19-21]. When the inner diameter of the blood vessel is larger than $3 \mathrm{~mm}$, the estimated

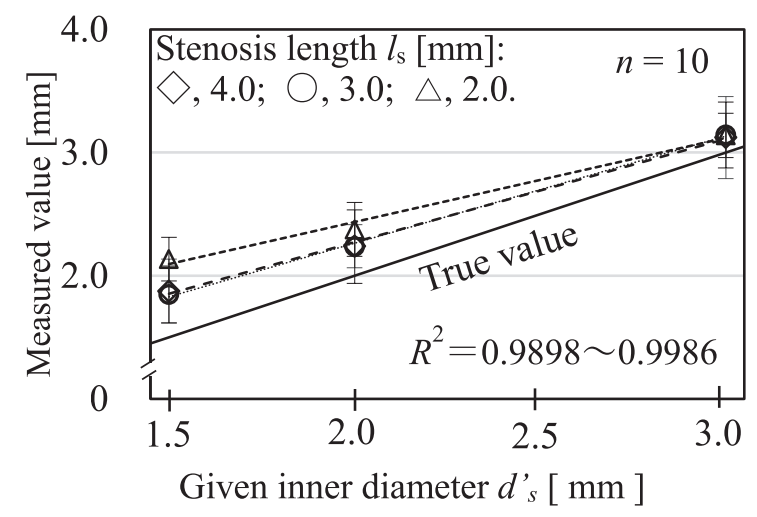

Fig. 10 Inner diameter of blood vessel model with localized stenosis measured using the proposed technique. diameter agreed well with the true value, with less than $5 \%$ error even without calibration. This result suggests great potential of the proposed technique for detection of stenotic change in blood vessels of the AV fistula.

\subsection{Detection of asymmetrical stenosis}

In practical applications related to an AV fistula, blood vessels often become narrow asymmetrically with respect to the vessel axis. This non-coaxial stenosis makes stenosis detection difficult because of increased scattering in the dominant volume in the image range of interest (ROI). To examine the applicability of the proposed technique, we attempted to detect the blood diameter change with a model phantom of non-coaxial stenosis. Figure 11 portrays the phantom structure. A portion of blood vessel $5 \mathrm{~mm}$ in diameter was made narrow over a 5-mm length along the blood vessel axis. The cross-section of the narrowed blood part was a circle shifted to one side of the intact blood vessel. Diameter of the narrowed portion was measured using the proposed technique in phantoms with non-coaxial stenoses of different diameters. Figure 12 portrays transillumination images obtained before and after deblurring. White dots represent the estimated positions of the blood-wall interface. Even in this difficult condition, the blood-wall boundary was detected correctly in the deblurred image. Figure 13 presents the measured diameters of the non-coaxial stenosis. The measured values were larger than the true diameters, with error as large as $40 \%$. This finding underscores the possibilities and limitations of our measurement system to handle severe blurring in transillumination images. However, the coefficient of determination was approximately unity. Therefore, we can expect that correct estimation can be achieved when using appropriate calibration. This result suggests that the proposed technique

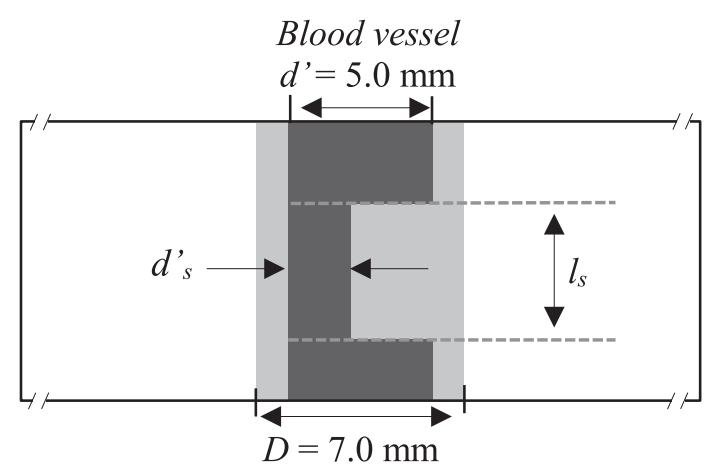

Fig. 11 Cross section of phantom structure to simulate localized non-coaxial stenosis: $d_{s}{ }_{s}$, inner diameter of stenotic portion; $l_{s}$, longitudinal length of stenotic portion; intact blood vessel inner diameter $d^{\prime}=$ $5.0 \mathrm{~mm}$; blood vessel outer diameter $D=7.0 \mathrm{~mm}$; blood vessel depth $t=1.0 \mathrm{~mm}$. 


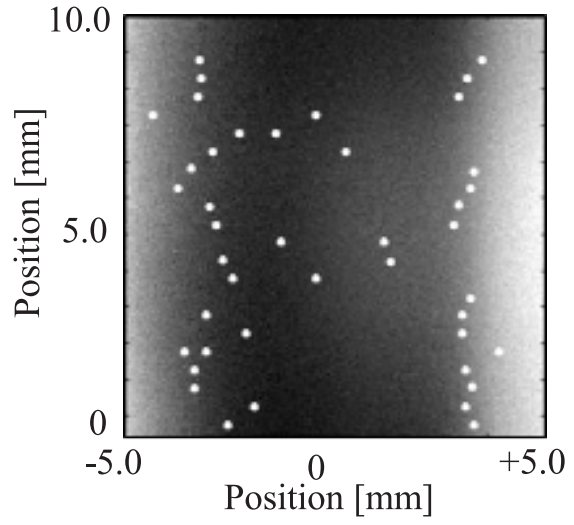

(a) Detection of blood boundary in original image

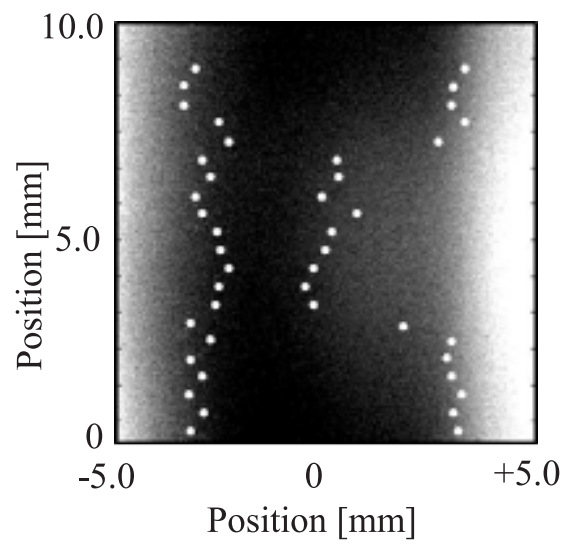

(b) Detection of blood boundary in deblurred image

Fig. 12 Detection of localized non-coaxial stenosis in transillumination imaging: outer diameter $D=7.0 \mathrm{~mm}$; inner diameter $d^{\prime}=5.0 \mathrm{~mm}$; inner diameter of stenotic portion $d^{\prime}{ }_{s}=2.0 \mathrm{~mm}$; length of stenosis $l_{s}=$ $5.0 \mathrm{~mm}$.

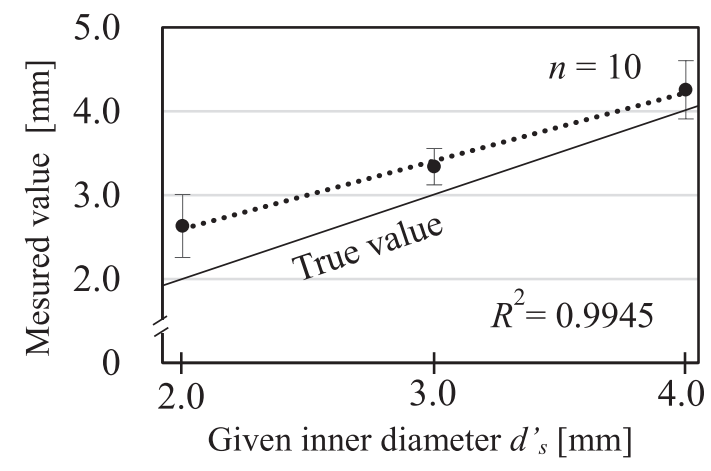

Fig. 13 Inner diameter of blood vessel model with localized non-coaxial stenosis measured by the proposed technique.

enables us to detect and measure localized non-coaxial stenoses in AV fistulae at an early stage.

\section{CONCLUSION}

A new optical imaging technique was proposed to realize noninvasive monitoring and management of an AV fistula for patients on chronic dialysis. Using NIR light diffusely scattered in the human body, transillumination imaging of the blood vessel in the AV fistula becomes possible. With light illumination from both sides of a human forearm, the subcutaneous blood vessel can be visualized even in thick human body tissues such as the adult forearm. An imaging system was constructed to examine the feasibility of the proposed technique. To measure the blood vessel diameter, we found that PSF deconvolution is effective to suppress blurring of a blood vessel image obtained even with strong scattering in interstitial tissues.

Validity of the proposed technique was confirmed through measurements using a blood vessel model 2.0$4.0 \mathrm{~mm}$ in diameter. Then, a model phantom was constructed to simulate a localized stenosis in the blood vessel. Using this phantom, the proposed technique to detect the stenosis in the $\mathrm{AV}$ fistula was evaluated. Although the vessel diameter was overestimated, change in vessel diameter of as small as $1.5-2.0 \mathrm{~mm}$ was detected sensitively. Applicability of the proposed technique to a more realistic case was demonstrated using a model phantom with a non-concentric stenosis. These results verified the validity and the promising potential of the proposed technique for the monitoring and management of the $\mathrm{AV}$ fistula for chronic dialysis patients.

The proposed technique can achieve noninvasive and quantitative inspection of the AV fistula using safe and simple apparatus. Because it requires no special skill or experience to use, it is useful not only in clinical applications but also in homecare treatment. It can contribute greatly to improvement of AV fistula management by recording the temporal changes in the transillumination image and the blood vessel inner diameter over a long period of time. Further improvement of the measurement accuracy and design of an instrument suitable for clinical use are tasks for future research.

\section{Acknowledgment}

This work was supported by JSPS KAKENHI Grant Number JP17K01418.

\section{References}

1. American Society of Clinical Oncology: Update of recommendations for the use of white blood cell growth factors: an evidence-based clinical practice guideline. J Clin Oncol. 24(19), 3187-3205, 2006.

2. Santoro D, Benedetto F, Mondello P, Pipitò N, Barillà D, Spinelli F, Ricciardi CA, Cernaro V, Buemi1 M: Vascular access for hemodialysis: current perspectives. Int J Nephrol Renovasc Dis. 


\section{7, 281-294, 2014}

3. Ghonemy TA, Farag SE, Soliman SA, Amin EM, Zidan AA: Vascular access complications and risk factors in hemodialysis patients: A single center study. Alexandria J Med. 52(1), 67-71, 2016.

4. Mendelson Y: Pulse oximetry: theory and applications for noninvasive monitoring. Clin Chem. 38(9), 1601-1607, 1992.

5. Key H, Jackson PC, Wells PNT: New approaches to transillumination imaging. J Biomed Eng. 10(2), 113-118, 1988.

6. Katra R: System and method for non-invasive monitoring of hematocrit concentration. U.S. Patent Application No 15/866, 118, 2019.

7. Wadhwani M, Sharma A, Pillai A, Pisal N, Bhowmick M: Vein Detection System using Infrared Light. Int J Sci Eng Res. 6(12), 780-786, 2015.

8. Miyake RK, Zeman HD, Duarte FH, Kikuchi R, Ramacciotti E, Lovhoiden G, Vrancken C: Vein imaging: a new method of near infrared imaging, where a processed image is projected onto the skin for the enhancement of vein treatment. Dermatol Surg. 32(8), 1031-1038, 2006.

9. Wang K, Ma H, Popoola OP, Li J: Finger vein recognition. Biometrics. 31-53, 2010.

10. Song W, Kim T, Kim HC, Choi JH, Kong HJ, Lee SR: A finger-vein verification system using mean curvature. Pattern Recognition Lett. 32(11) 1541-1547, 2011.

11. Jacques SL: Origins of tissue optical properties in the UVA, visible, and NIR regions. OSA TOPS on Advances in Optical Imaging and Photon Migration vol. 2, pp. 364-369, 1996.

12. Tran TN, Yamamoto K, Namita T, Kato Y, Shimizu K: Three-dimensional transillumination image reconstruction for small animal with new scattering suppression technique. Biomed Opt Express. 5(5), 1321-1335, 2014.

13. Tuchin VV: Tissue Optics, Light Scattering Methods and Instruments for Medical Diagnostics, Third Edition, SPIE Press Book, 2015.

14. Boas DA, Pitris C, Ramanujam N: Handbook of Biomedical Optics CRC Press, 2011

15. Vo-Dinh T. Biomedical Photonics Handbook: Biomedical Diagnostics, CRC Press, 2014.

16. Duan J, Tench C, Gottlob I, Proudlock F, Bai L: Optical coherence tomography image segmentation. In: 2015 IEEE International Conference on Image Processing (ICIP). IEEE, pp. 42784282, 2015.

17. Shimizu K, Tochio K, Kato Y: Improvement of transcutaneous fluorescent images with a depth-dependent point-spread function. Appl Opt. 44(11), 2154-2161, 2005.

18. The Japanese Society for Dialysis Therapy: Guidelines of Vascular Access Construction and Repair for Chronic Hemodialysis. J Jpn Soc Dialysis Ther. 44(9), 855-937, 2011.

19. Radosa CG, Radosa JC, Weiss N, Schmidt C, Werth S, Hofmockel T, Plodeck V, Gatzweiler C, Laniado M, Hoffmann RT: Endovascular creation of an arteriovenous fistula (endoAVF) for hemodialysis access: first results. Cardiovasc Interventional Radiol. 40(10), 1545-1551, 2017.

20. Smith GE, Gohil R, Chetter IC: Factors affecting the patency of arteriovenous fistulas for dialysis access. J Vasc Surg. 55(3), 849-855, 2012.

21. Wong V, Ward R, Taylor J, Selvakumar S, How T. V, Bakran A:
Factors associated with early failure of arteriovenous fistulae for haemodialysis access. Eur J Vasc Endovasc Surg. 12(2), 207213, 1996.

\section{Hideaki KaMiYama}

Hideaki KamiYAMA received B.E. and M.E. (2018) degrees from Hokkaido University of Science, Sapporo, Japan. He is currently a Ph.D. student of Graduate school of Hokkaido University of Science. His research interests include optical measurement in biomedical engineering. He is a mem-

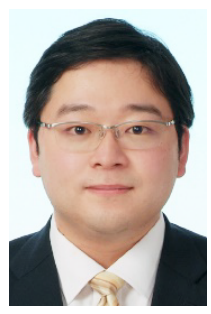
ber of IEEE and JSMBE. He received IEEE Sapporo Section Student Paper Contest Encouraging Prize (2017) and Encouraging Prize from Hokkaido Branch of Japanese Society for Medical and Biological Engineering (2018).

\section{Masataka KitAmA}

Masataka KiTAMA received his Ph.D. degree in Engineering from Hokkaido University, Sapporo, Japan (1995). He was a JSPS Research Fellowship for Young Scientists (1994-96). He has been a Lecturer, an Associate and a Professor of Department of Applied Electronics in Hokkaido Univer-

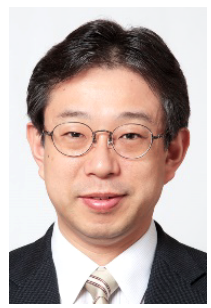
sity of Science (former Hokkaido Institute of Technology), Sapporo, Japan since 1996. He is currently a professor of the Graduate School of Health Sciences at Hokkaido University of Science. He has been engaged in the studies of biomedical engineering including those of wave propagation in biological media, optical measurement and non-invasive measurement. He is a member of IEICE, JSMBE and JSMI. He received Research Promotion Award (1992) and Best Paper Award (1995) from Japan Society for Medical and Biological Engineering.

\section{Hisae Odagiri-Shimizu}

Hisae Odagiri-Shimizu received her Ph.D. degree from Hokkaido University, Sapporo, Japan, in 2002. She is currently a professor of Department of Clinical Engineering, Hokkaido University of Science, Sapporo, Japan. She has been engaged in studies of biomedical engineering, particularly the

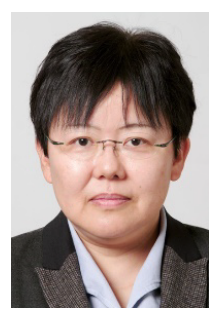
biological effects of electromagnetic fields. She is a member of IEEE, Japanese Society for Medical and Biological Engineering, and the Institute of Electronics Information and Communication Engineers. 


\section{Masaji Yamashita}

Masaji YAMASHITA received his Ph.D. degree in Engineering from Hokkaido University (Japan) in 1988. He is a Professor of Clinical Engineering at Faculty of Health Sciences, Hokkaido University of Science (Japan) since 2004, after having contributed for 13 years as an Associate Professor of

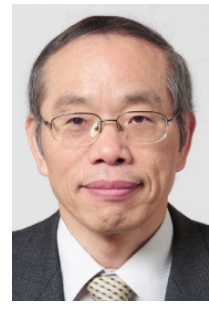
Biomedical Engineering and Electric Engineering to the Faculty of Engineering at Hokkaido Institute of Technology (Japan) since 1991. His research interests are biomedical signal processing, emotion, biomedical telemetry and bio-electromagnetics. He is a member of Japanese Society for Medical and Biological Engineering, Japan Society of Kansei Engineering, Japan Society of Physiological Anthropology, Institute of Electronics, Information and Communication Engineers, and Society of Instrument and Control Engineers.

\section{Kojima Yонгсніro}

Kojima Yoнгсніro He received his BE, ME and Dr. Eng. degree, all in applied electronics engineering, from Hokkaido Institute of Technology in 1990, 1992 and 1995, respectively. He is now a Professor at Department of Electrical and Electronic Engineering, Faculty of Engineering of Hokkaido Uni-

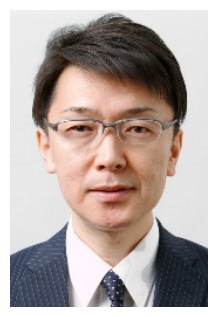
versity of Science. His main research interests are focused on development of sensor engineering. Dr. Kojima is a member of the Institute of Electrical Engineers of Japan and the Institute of Industrial Applications Engineers.

\section{Koichi SHIMIZU}

Koichi SHIMIZu received M.S. (1976) and Ph.D. (1979) degrees, from University of Washington, Seattle, USA. He was Research Associate in University of Washington 1974-79. He was an Assistant-, an Associate- Professors, and a Professor in Hokkaido University, Sapporo, Japan in 1979-

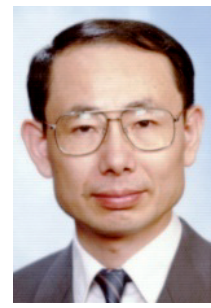
2016. He is currently a Professor Emeritus of Hokkaido University and a Professor of Waseda University, Kitakyushu, Japan. He has been engaged in the studies of biomedical engineering including those of wave propagation in biological media, optical measurement, biotelemetry and biological effects of electromagnetic field. He served as an associate editor of IEEE Trans. ITB in 1999-2007. He has been a Fellow of the Electromagnetics Academy, and an editorial board member of Scientific Reports, Nature. He is a member of IEEE, OSA, ISOB, IEEJ, IEICE and JSMBE. He received Research Promotion Award (1981), Best Paper Award (1995) and New Technology Development Award (2020) from Japan Society for Medical and Biological Engineering. 